\title{
Estabilidade oxidativa e perfil de ácidos graxos do leite de vacas suplementadas com óleo de linhaça na dieta associado ou não ao selenito de sódio injetável
}

\author{
[Oxidative stability and fatty acid profile content of milk from cows fed linseed oil diet with \\ or without injectable sodium selenite] \\ L. Cardozo, M. Cecim", E. Soares,D.K. Moreira, R. Schuster, N.S.P.S. Richards, \\ T.C. Unfer, A. Quatrin, G. Fuke, M. Roehrs \\ Universidade Federal de Santa Maria - Santa Maria, RS
}

\begin{abstract}
RESUMO
Avaliou-se o efeito da suplementação com óleo de linhaça na dieta, com ou sem injeção de selenito de sódio, sobre o perfil de ácidos graxos e sobre a estabilidade oxidativa do leite de vacas leiteiras. Catorze vacas foram distribuídas em três tratamentos: as do grupo 1, que receberam diariamente $400 \mathrm{~mL}$ de óleo de linhaça (LIN); as do grupo 2, 400mL de óleo de linhaça + 0,2mg/kg de selenito de sódio IM (LINSe); e as do grupo 3 (controle), que não foram tratadas (C). O óleo foi fornecido diariamente após 15 dias da aplicação única de selenito de sódio, e o experimento teve duração de quatro semanas. Os animais suplementados com o óleo de linhaça produziram leite com altas porcentagens de ácido linoleico conjugado (CLA) e de ômega 3, contudo mais suscetível à oxidação. A aplicação injetável de selenito de sódio mostrou-se eficaz ao impedir a oxidação prematura do leite. Concluiu-se que a inclusão do óleo de linhaça na dieta de vacas leiteiras resulta em aumento do CLA, do ômega 3 e, consequentemente, da oxidação do leite, necessitando, assim do uso de substâncias antioxidantes ou promotores antioxidantes, como o selenito de sódio injetável.
\end{abstract}

Palavras-chave: antioxidante, ácido linoleico conjugado, selenito de sódio, glutationa peroxidase, TBARS

\begin{abstract}
The aim of the present study was to evaluate the effect of dietary linseed oil supplementation with or without injectable sodium selenite on the fatty acid profile and oxidative stability of milk from dairy cows. Fourteen cows were allocated into four treatment groups: Group 1 which received 400mL of linseed oil daily (LIN); Group 2, 400mL of linseed oil + 0,2mg/BW sodium selenite IM (LINSe); Group 3, untreated controls $(C)$. The oil was supplied daily after 15 days of a single application of sodium selenite and during 4 weeks. Animals supplemented with linseed oil produced milk with higher levels of conjugated linoleic acid (CLA) and omega 3, but were also more susceptible to oxidation. The application of sodium selenite was effective to prevent premature oxidation of milk. We concluded that the supplementation of a cow's diet with linseed oil promoted increased CLA and omega 3 in milk, which in turn becomes more susceptible to oxidation, requiring the use of antioxidants or promoters of antioxidant activity such as injectable sodium selenite.
\end{abstract}

Keywords: oxidative stability, conjugated linoleic acid, sodium selenite, glutathione peroxidase, TBARS

\section{INTRODUÇÃO}

O enriquecimento do leite bovino com moléculas nutracêuticas, como os ácidos graxos ômega e o ácido linoleico conjugado (CLA), é uma

Recebido em 17 de outubro de 2011

Aceito em 4 de dezembro de 2012

*Autor para correspondência (corresponding author)

E-mail: mcecim@smail.ufsm.br tendência atual e pode ser obtido com o fornecimento de gordura na dieta dos animais em lactação. Gorduras ricas em ômega 3 ou ácido linolênico (cis9, cis12, cis15-C18:3), quando adicionadas à dieta, promovem aumento do CLA, já que o ômega 3 é precursor da síntese 
desse ácido graxo durante o processo de biohidrogenação ruminal (Harfoot e Hazlewood, 1997), que é um ato de defesa dos microorganismos do rúmen contra as gorduras insaturadas que lhes são tóxicas (Jenkins, 1993).

Modificações nas características dietéticas causam alterações no metabolismo ruminal. Apesar disso, a inclusão de fontes de óleo na dieta de ruminantes é uma alternativa já utilizada para o atendimento às exigências de energia de animais de alta produção leiteira. $\mathrm{O}$ uso de lipídios, tanto de origem animal quanto vegetal, é ainda motivo de contradições, pois $o$ conhecimento mantém-se restrito quanto aos níveis e às formas de inclusão (protegidas ou não) e dos seus efeitos no consumo (NRC, 2001). A maioria dos trabalhos confirma que o uso de lipídios na alimentação exerce pouco ou nenhum efeito sobre as atividades da flora microbiana e as demais características do ambiente ruminal, desde que não ultrapasse o valor de $7 \%$ da matéria seca total da dieta.

A linhaça é uma oleaginosa que apresenta $32 \%$ de gordura, com $60 \%$ dos ácidos graxos considerados poli-insaturados, rica em ômega 3, podendo, assim, aumentar a concentração de CLA no leite dos animais durante a biohidrogenação dele até o ácido graxo trans11 C18:1, quando usada na dieta (Ward et al., 2002).

A modificação no perfil de ácidos graxos do leite pode alterar várias propriedades físicas e químicas da gordura, como a firmeza, o ponto de fusão, a viscosidade, a estabilidade oxidativa e o "flavor" (sabor e/ou odor). Sabe-se que os lipídios do leite são protegidos contra a oxidação por antioxidantes naturais enzimáticos e não enzimáticos (Lindmark-Mansson e Akesson, 2000). Dentre as enzimas antioxidantes, destacase a glutationa peroxidase (GSHPx), uma enzima selênio-dependente que atua metabolizando os hidroperóxidos (Surai, 2006).

Durante a decomposição dos peróxidos de hidrogênio, formam-se os produtos secundários da oxidação, vários dos quais são voláteis e responsáveis pela formação de odores e/ou gostos indesejáveis no produto (LindmarkMansson e Akesson, 2000).
A atividade da GSHPx depende da biodisponibilidade do selênio (Surai, 2006). Focos de deficiência de selênio têm sido identificados em várias regiões do mundo, assim como no Brasil (Moraes et al., 1999), tornando indispensável à suplementação aos animais.

Para que o selênio seja incorporado especificamente em selenoproteínas funcionais, como a GSHPx, é necessário que as formas orgânicas e inorgânicas sejam reduzidas a seleneto, e este metabolizado a selenocisteína (SeCis). Para tal, o seleneto reage com ATP, formando selenofosfato, numa reação catalisada pela selenofosfato sintetase. Junto a um resíduo de serina, o selenofosfato, forma uma SeCis, que é pós-translacionalmente inserida nas selenoproteínas funcionais através de um códon UGA específico (Sunde e Hoekstra, 1980; Driscoll e Copeland, 2003).

Para fugir da rota metabólica extensa que o selênio suplementado via oral necessita percorrer, Oblitas et al. (2000) testaram, em animais deficientes em selênio, uma dose de $5 \mathrm{mg} / 100 \mathrm{~kg}$ de peso de selenito de sódio injetável e mediram o efeito na atividade da enzima glutationa peroxidase sanguínea e no ganho de peso dos animais. Concluiu-se, assim, que essa dose produz um incremento na atividade da glutationa peroxidase dos 30 aos 90 dias após a aplicação.

Dessa forma, o objetivo deste trabalho foi avaliar o efeito da suplementação com óleo de linhaça na dieta de vacas leiteiras, associado ou não ao selenito de sódio injetável, sobre o perfil de ácidos graxos e a estabilidade oxidativa do leite.

\section{MATERIAL E MÉTODOS}

O experimento foi realizado em um rebanho leiteiro localizado na cidade de Novo Cabrais, RS, durante os meses de abril e maio de 2010. Foram utilizadas 14 vacas leiteiras das raças Holandês e Jersey, de 60 a 240 dias em lactação, com produção média de $16,25 \mathrm{~kg}$ de leite por dia, distribuídas em blocos ao acaso, de forma uniforme em relação à raça, ao período e ao número de lactações, entre os grupos. A ordenha foi realizada duas vezes ao dia (5 e 16h) e medidas gerais de higiene foram adotadas. 
Os tratamentos utilizados no experimento foram: dieta com inclusão de $400 \mathrm{~mL}$ de óleo de linhaça (LIN); dieta com inclusão de $400 \mathrm{~mL}$ de óleo de linhaça $+0,2 \mathrm{mg} / \mathrm{kg}$ de $\mathrm{Na} 2 \mathrm{SeO} 3$ injetável (LINSe); e controle (C). O selenito de sódio foi aplicado via intramuscular (solução aquosa) no dia zero, o fornecimento de óleo iniciou no dia 15 e as coletas de leite ocorreram no dia 30 do experimento. O período experimental teve duração de quatro semanas. A dose diária de óleo de linhaça foi medida e misturada a $300 \mathrm{~g}$ de farelo de trigo, fornecida uma vez ao dia, após a ordenha da tarde, misturada à ração dos animais, para garantir consumo total. Amostras do concentrado e da silagem foram enviadas para análise laboratorial, e os resultados encontram-se na Tab. 1.

Tabela 1. Composição química dos alimentos fornecidos durante o período experimental

\begin{tabular}{cccc}
\hline & Ração & Farelo trigo & Silagem milho \\
\hline MS & 88,35 & 89,57 & 28,8 \\
MST & 88,35 & 89,57 & 94,7 \\
CZ (\%MS) & 9,24 & 3,53 & 4,49 \\
CZ (\%AI) & 8,17 & 3,17 & 1,29 \\
PB (\%MS) & 24,82 & 16,06 & 5,61 \\
PB (\%AI) & 21,93 & 14,39 & 1,61 \\
FDNc (\%MS) & 25,7 & 28,76 & 46,7 \\
FDNc (\%AI) & 22,7 & 25,76 & 13,45 \\
FDAc (\%MS) & 10,49 & 11,41 & 25,53 \\
FDAc (\%AI) & 9,27 & 10,22 & 7,35 \\
EE (\%MS) & 5,75 & 2,84 & 3,92 \\
EE (\%AI) & 5,08 & 2,54 & 1,13 \\
\hline
\end{tabular}

MS (matéria seca); MST (matéria seca total); CZ (cinzas); AI (amostra integral); PB (proteína bruta); FDNc (fibra em detergente neutro corrigida para cinzas); FDAc (fibra em detergente ácido corrigida para cinzas); EE (extrato etéreo).

Foram determinados os teores de matéria seca total (MST), matéria mineral (MM), proteína bruta $(\mathrm{PB})$ e extrato etéreo (EE), todos segundo a AOAC (1995); fibra em detergente ácido corrigida para cinzas (FDAc) e fibra em detergente neutro corrigida para cinzas (FDNc), conforme Van Soest et al. (1991), com adição de alfa-amilase termoestável mas sem uso de sulfito.

No levantamento do perfil de ácidos graxos, as amostras de leite foram coletadas no dia 30 do período experimental, durante a ordenha da manhã, de cada animal proporcionalmente, de modo a compor uma amostra homogênea de cada grupo. Foram utilizados tubos falcon de $50 \mathrm{~mL}$, sem adição de conservantes, e as amostras foram congeladas a $-20^{\circ} \mathrm{C}$. A extração dos lipídios das amostras de leite foi feita segundo a metodologia de Blight e Dyer (1959). Para a análise dos ácidos graxos, uma alíquota do extrato lipídico, contendo aproximadamente $100 \mathrm{mg}$ de lipídios, foi seca em evaporador rotatório e transmetilada de acordo com o método de Hartman e Lago (1973). A identificação dos ácidos graxos foi realizada por meio da comparação do tempo de retenção dos ácidos graxos das amostras com o de padrões conhecidos. O teor de cada ácido graxo nas amostras de leite foi calculado de acordo com a área de cada um dos picos obtidos nos cromatogramas, sendo estas áreas multiplicadas por 100 e o valor resultante, dividido pelo total de ácidos graxos da amostra.

Análises de reações com ácido tiobarbitúrico (TBARS), que identifica os produtos secundários da peroxidação lipídica, foram realizadas imediatamente após a chegada ao laboratório das amostras, aproximadamente oito horas após a coleta de leite, 24 e 96 horas depois da primeira análise. Foram coletados $100 \mathrm{~mL}$ de leite de cada grupo experimental para cada tempo de análise, e a metodologia utilizada foi a mesma descrita por Paschoal et al. (2007). Para avaliação da atividade da enzima glutationa peroxidase, utilizou-se metodologia adaptada de Paglia e Valentine (1967).

As análises de perfil de ácidos graxos, de reações com ácido tiobarbitúrico (TBARS) e da atividade da glutationa peroxidase foram submetidas à análise de variância, sendo utilizado o teste de Tukey (significância de $\mathrm{P}<0,05$ ) para comparar as médias testadas. As análises foram realizadas 
com auxílio do software Graph Pad InStat 3, versão 3.10 .

\section{RESULTADOS E DISCUSSÃO}

Com a inclusão diária de $400 \mathrm{~mL}$ de óleo de linhaça na alimentação, os animais não apresentaram distúrbios metabólicos perceptíveis nem diminuição considerável no consumo, mesmo sendo o tempo de adição de óleo na dieta do presente estudo considerado adaptativo. Segundo Benson et al. (2001), a utilização de fontes lipídicas conhecidamente ricas em ácidos graxos poli-insaturados e não esterificados pode influenciar o consumo de matéria seca. Entretanto, Kelly et al. (1998) avaliaram fontes de óleos vegetais ricas em ácidos graxos poliinsaturados (oleico, linoleico e linolênico) na dieta de vacas leiteiras e verificaram que a ingestão de matéria seca não foi alterada pela fonte de lipídio adicionada à dieta.

Para Loor et al. (2002), a suplementação da dieta de vacas leiteiras com lipídios protegidos e óleo de canola não afetou a digestibilidade aparente da MS, PB e FDA no trato digestório total. Além disso, a concentração de nitrogênio amoniacal do rúmen diminuiu em resposta à suplementação lipídica, sendo menor para as fontes protegidas. Estudos similares também indicaram diminuição da concentração de nitrogênio amoniacal quando uma dieta controle foi suplementada com óleo de linhaça ((Igwuegbu e Sutton, 1982) e óleo de canola (Doreau e Ferlay et al., 1995).

Os ácidos graxos de cadeia longa (C18), apresentados na Tab. 2, estavam aumentados no grupo LINSe em relação ao grupo LIN e ao controle, o que não foi observado nos trabalhos de Ramaswamy et al. (2001) e Paschoal et al. (2007). Porém, os valores aumentados (1,06 e $1,26 \mathrm{~g} / 100 \mathrm{~g}$ ) obtidos neste trabalho em relação à concentração de CLA nos grupos tratados com óleo são semelhantes aos observados por Ramaswamy et al. (2001), em vacas alimentadas com forragem conservada e alta concentração de óleo vegetal, e por Paschoal et al. (2007), que utilizaram soja extrusada.

Tabela 2. Perfil de ácidos graxos do leite de vacas que receberam óleo de linhaça (LIN); óleo de linhaça + selênio injetável (LINSe) e do grupo-controle (C)

\begin{tabular}{|c|c|c|c|c|}
\hline Perfil & $\begin{array}{l}\mathrm{LIN} \\
\mathrm{n}=5\end{array}$ & $\begin{array}{c}\text { LINSe } \\
n=5\end{array}$ & $\begin{array}{c}C \\
n=4\end{array}$ & $p$ \\
\hline $\mathrm{C} 4: 0$ & $16,02 \pm 0,01 \mathrm{a}$ & $0,51 \pm 0,01 b$ & $13,10 \pm 0,01 \mathrm{c}$ & $<0.0001$ \\
\hline C6:0 & $0,56 \pm 0,01 \mathrm{a}$ & $0,58 \pm 0,01 \mathrm{a}$ & $0,77 \pm 0,01 b$ & $<0.0001$ \\
\hline C8:0 & $0,54 \pm 0,01 \mathrm{a}$ & $0,52 \pm 0,01 \mathrm{a}$ & $0,69 \pm 0,01 b$ & $<0.0001$ \\
\hline C10:0 & $1,77 \pm 0,01 \mathrm{a}$ & $1,73 \pm 0,01 b$ & $2,11 \pm 0,01 \mathrm{c}$ & $<0.0001$ \\
\hline C11:0 & $0,24 \pm 0,01 \mathrm{a}$ & $0,24 \pm 0,01 \mathrm{a}$ & $0,28 \pm 0,01 b$ & $<0.0039$ \\
\hline C12:0 & $2,82 \pm 0,01 \mathrm{a}$ & $2,82 \pm 0,01 \mathrm{a}$ & $3,09 \pm 0,01 \mathrm{~b}$ & $<0.0001$ \\
\hline C14:0 & $9,47 \pm 0,01 \mathrm{a}$ & $9,85 \pm 0,01 b$ & $10,78 \pm 0,01 \mathrm{c}$ & $<0.0001$ \\
\hline C14:1n & $1,06 \pm 0,01 \mathrm{a}$ & $1,10 \pm 0,01 b$ & $0,98 \pm 0,01 \mathrm{c}$ & $<0.0001$ \\
\hline C15:0 & $0,92 \pm 0,01 \mathrm{a}$ & $1,15 \pm 0,01 \mathrm{~b}$ & $1,01 \pm 0,01 \mathrm{c}$ & $<0.0001$ \\
\hline C16:0 & $30,51 \pm 0,01 \mathrm{a}$ & $32,69 \pm 0,01 b$ & $31,37 \pm 0,01 \mathrm{c}$ & $<0.0001$ \\
\hline C16:1 & $1,71 \pm 0,01 \mathrm{a}$ & $1,97 \pm 0,01 b$ & $1,34 \pm 0,01 \mathrm{c}$ & $<0.0001$ \\
\hline C17:0 & $0,67 \pm 0,01 \mathrm{a}$ & $0,77 \pm 0,01 b$ & $0,78 \pm 0,01 b$ & $<0.0001$ \\
\hline C18:0 & $8,06 \pm 0,01 \mathrm{a}$ & $12,23 \pm 0,01 b$ & $9,47 \pm 0,01 \mathrm{c}$ & $<0.0001$ \\
\hline C18:1n9T & $3,52 \pm 0,01 \mathrm{a}$ & $4,36 \pm 0,01 b$ & $1,94 \pm 0,01 \mathrm{c}$ & $<0.0001$ \\
\hline VACÊNICO & $0,40 \pm 0,01 \mathrm{a}$ & $1,63 \pm 0,01 b$ & $0,40 \pm 0,01 \mathrm{a}$ & $<0.0001$ \\
\hline C18:1n9C & $17,87 \pm 0,01 \mathrm{a}$ & $22,9 \pm 0,01 b$ & $18,69 \pm 0,01 \mathrm{c}$ & $<0.0001$ \\
\hline $\mathrm{C} 18: 2 \mathrm{n} 6 \mathrm{C}$ & $2,31 \pm 0,01 \mathrm{a}$ & $3,17 \pm 0,01 b$ & $2,05 \pm 0,01 \mathrm{c}$ & $<0.0001$ \\
\hline C18:2C9T11 CLA1 & $1,06 \pm 0,01 \mathrm{a}$ & $1,26 \pm 0,01 b$ & $0,86 \pm 0,01 \mathrm{c}$ & $<0.0001$ \\
\hline C18:3 & $0,49 \pm 0,01 \mathrm{a}$ & $0,51 \pm 0,01 \mathrm{a}$ & $0,27 \pm 0,01 b$ & $<0.0001$ \\
\hline
\end{tabular}

Resultados expressos em média \pm SD. Significância $\mathrm{P}<0,05$. Letras diferentes na mesma linha significam diferenças identificadas pelo teste Tukey. 
Observou-se também que, em trabalhos que utilizaram grãos de soja triturados (Santos et al., 2001) e soja extrusada (Paschoal et al., 2007), as concentrações de CLA foram numericamente mais baixas que as encontradas neste estudo. Isso demonstra que a maior concentração desse ácido graxo é obtida utilizando-se o óleo e não os grãos de oleaginosas, mesmo quando estes sofrem processo térmico ou mecânico. Segundo esses autores, a baixa concentração de CLA poderia estar relacionada à baixa disponibilidade dos lipídios à bio-hidrogenação, uma vez que estes estariam presos à matriz proteica das sementes.

No presente estudo, o ômega 3 ou ácido linolênico também apresentou altas concentrações nos animais tratados com óleo de linhaça em relação aos do grupo-controle (Tab. 2). Paschoal et al. (2007) não observaram diferenças entre os grupos tratados e o controle em relação à concentração desse ácido graxo. A Tab. 2 mostra que houve alteração no perfil lipídico do leite e, consequentemente, aumento da concentração de ácidos graxos poliinsaturados, mais suscetíveis à oxidação, o que é confirmado pelos valores de TBARS no tempo 96 da análise (Fig. 1), em que o grupo LIN diferiu significativamente dos demais, mostrando-se mais oxidado, e o grupo LINSe manteve-se semelhante ao controle, indicando possível efeito do selênio como promotor do efeito antioxidante observado. Paschoal et al. (2007) observaram, em animais suplementados com soja extrusada e selênio orgânico oral, o mesmo aumento na oxidação no tempo 96 de análise e maior oxidação no leite dos animais que receberam dieta lipídica em relação aos que a receberam e foram suplementados com selênio.

Oblitas et al. (2000) citaram incremento da atividade sanguínea da glutationa peroxidase, enzima antioxidante dependente de selênio, em animais que receberam dose injetável de selenito de sódio a partir de 30 dias de sua aplicação. Stagsted (2006) não detectou qualquer atividade da glutationa peroxidase (GSHPx) no leite bovino, diferentemente do resultado de Hojo (1982), que detectou atividade da enzima em leite cru. O primeiro autor ainda citou que a presença e a importância da glutationa peroxidase para a estabilidade oxidativa do leite de vaca não é clara, e, para Phipps et al. (2008), embora GSHPx seja uma importante enzima dependente de selênio no leite, outros compostos também apresentam atividade antioxidante, o que justifica os resultados inexpressivos da atividade da enzima encontrados no grupo tratado com selênio, apesar de a oxidação ter sido retardada nesse grupo (Fig. 2).

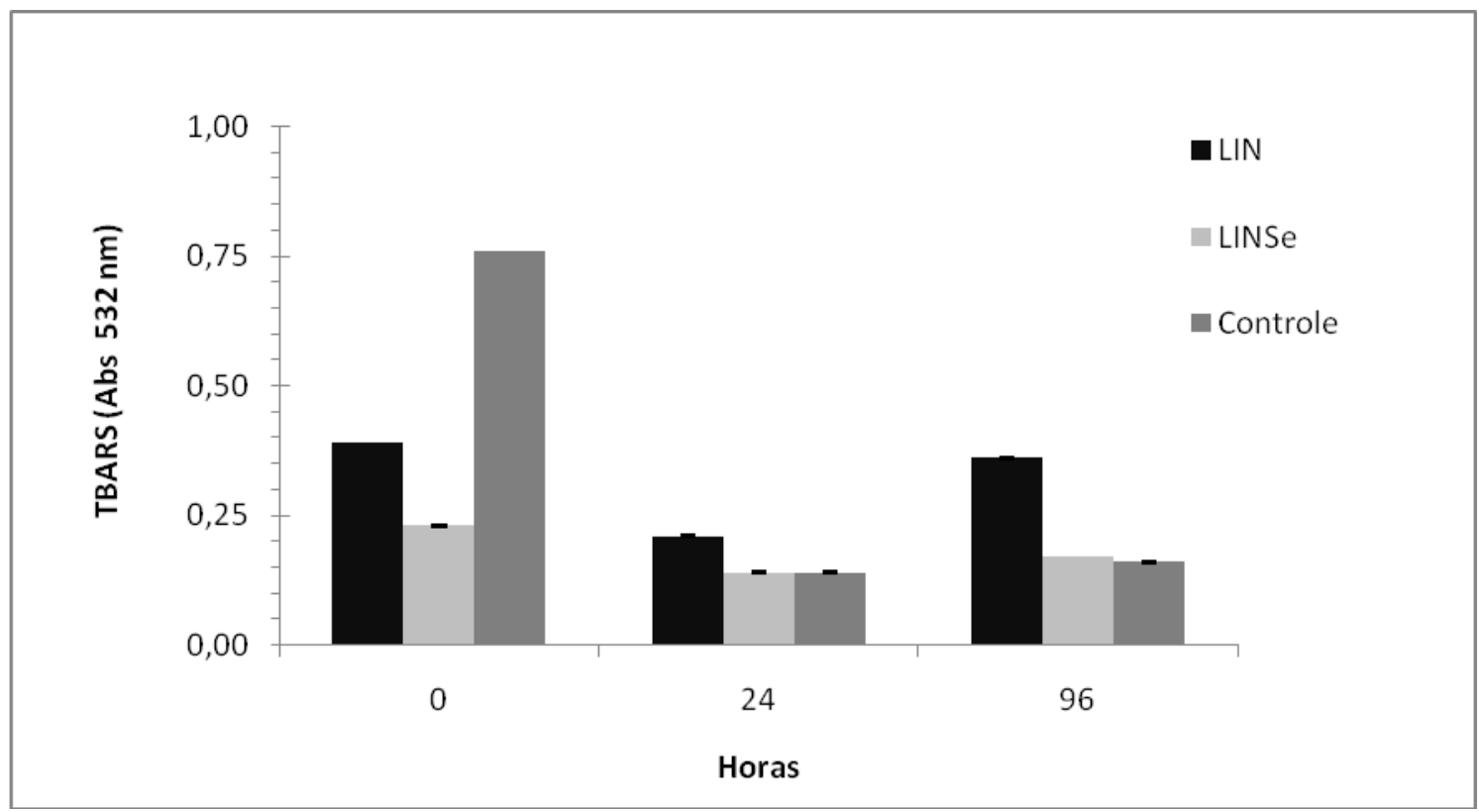

Figura 1. Análises de reações com ácido tiobarbitúrico (TBARS) zero, 24 e 96 horas após a coleta do leite de vacas que receberam óleo de linhaça (LIN); óleo de linhaça + selênio injetável (LINSe) e do grupo-controle $(\mathrm{C})$. 


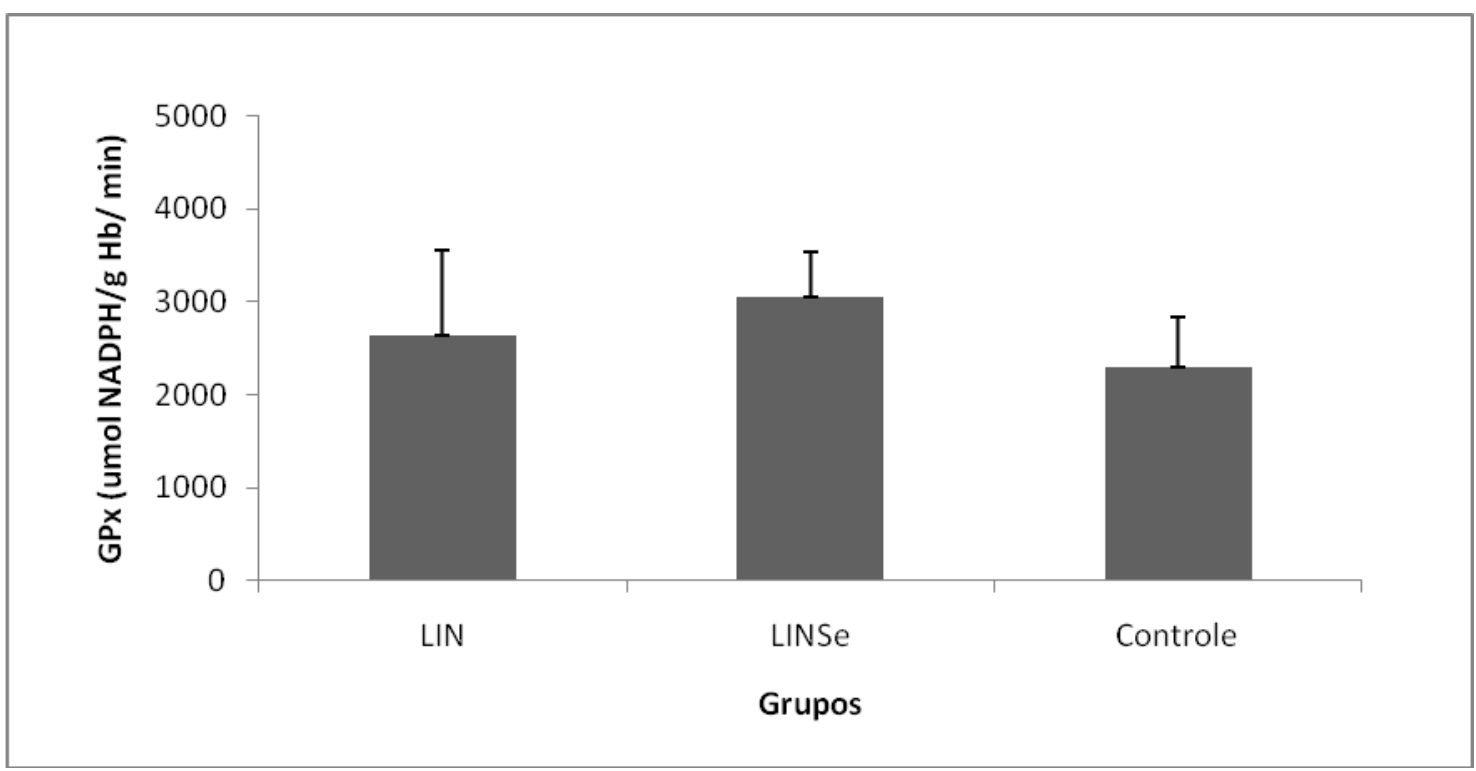

Figura 2. Atividade da enzima glutationa peroxidase do leite de vacas que receberam óleo de linhaça (LIN); óleo de linhaça + selênio injetável (LINSe) e do grupo-controle (C).

O presente estudo demonstrou que animais que apresentaram o perfil de ácidos graxos do leite modificado pela adição de óleo de linhaça na dieta e receberam uma dose injetável de selenito de sódio não diferiram significativamente dos animais do grupo-controle em relação aos valores de TBARS 24 e 96 horas após a coleta do leite. Além disso, a atividade da GSHPx não foi significativamente diferente entre os grupos (Fig. 2), demonstrando, portanto, que uma aplicação de selenito de sódio, na dose de $0,2 \mathrm{mg} / \mathrm{kg}$, favorece a estabilidade oxidativa do leite após 30 dias, porém essa atividade antioxidante não se deve especificamente à enzima GSHPx.

\section{CONCLUSÕES}

O uso da suplementação com óleo de linhaça na dieta de vacas leiteiras resulta em leite e produtos lácteos com valor nutracêutico agregado. Entretanto, esses produtos apresentam maior sensibilidade à oxidação, prejudicando, assim, as características sensoriais.

\section{AGRADECIMENTOS}

Pelo fornecimento do óleo de linhaça, à empresa Farinhas Integrais Cisbra Ltda.; aos colaboradores na coleta e no processamento das análises: Carlos Rubim, Rudolf Scheibler e Andreane Filappi; à CAPES, ao CNPQ e ao
PPGMV-UFSM, pelo apoio financeiro. Este trabalho foi aprovado pela Comissão de Ética em Pesquisa da Universidade Federal de Santa Maria, sob o número 88/2009, e os estudos foram realizados de acordo com normas éticas.

\section{REFERÊNCIAS}

AOAC - ASSOCIATION OF OFFICIAL ANALYTICAL CHEMISTS. Official Methods of Analysis. 12th ed. Washington, 1995.

BENSON, J.A.; REYNOLDS, C.K.; HUMPHRIES, D.J. et al. Effects of abomasal infusion of long-chain fatty acids on intake, feeding behavior and milk production in dairy cows. J. Dairy Sci., v.84, p.11821191, 2001.

BLIGH, E.G.; DYER, W.J. A rapid method of total lipid extraction and purification. Canadian $J$. Biochemistry Physiol., v.37, p.911-917, 1959.

DOREAU, M.; FERLAY, A. Effect of dietary lipids on the ruminal metabolism in the rumen: a review. Livestock Prod. Sci., v.43, p.97-110, 1995.

DRISCOLL, D.M.; COPELAND, P.R. Mechanism and regulation of selenoprotein synthesis. Annual Rev. of Nut., v.23, p.17-40, 2003.

HARFOOT, C.G.; HAZLEWOOD, G.P. Lipid metabolism in the rumen. In: HOBSON, P.N.; STEWART, C.S. (Eds.). The Rumen Microbial Ecosystem. 2.ed. Blackie Academic \& Professional: Great Britain, 1997. p.382-419. 
HARTMAN, L.; LAGO, R.C.A. Rapid preparation of fatty acid methyl esters from lipids. Lab. Practice, v.22, p.475-476, 1973

HOJO, Y. Selenium concentration and glutathione peroxidase activity in cow's milk. Biol. Trace Elem. Rese., v.4, p.233-239, 1982.

IGWUEGBU, O.A.; SUTTON, J.D. The effect of varying the amount of linseed oil supplementation on rumen metabolism in sheep. British J. Nutrit., v.48, p.365-375, 1982

JENKINS, T.C. Lipid metabolism in the rumen. J. Dairy Sci., v.76, p.3851-3863, 1993.

KELLY, M.L.; BERRY, J.R.; DWYER, D.A. et al. Dietary fatty acid sources affect conjugated linoleic acid concentrations in milk from lactating dairy cows. J. Nutrition, v.128, p.881-885, 1998.

LINDMARK-MANSSON, H.; AKESSON, B. Antioxidative factors in milk. British J. Nutrit., v.84, p.103-110, 2000 .

LOOR, J.J.; HERBEIN, J.H.; JENKINS, T.C. Nutrient digestion, biohydrogenation, and fatty acid profiles in blood plasma and milk fat from lactating Holstein cows fed canola oil or canolamide. Anim. Feed Sci. Technol., v.97, p.65-82, 2002

MORAES, S.S.; TOKARNIA, C. H.; DÖBEREINER, J. Microelement deficiencies and imbalances in cattle and sheep in some regions of Brazil. Pesq. Vet. Bras., v.19, p.19-33, 1999.

NUTRIENT requirements of dairy cattle - NRC. 7.ed. Washington: National Academy of Science, 2001. 385p.

OBLITAS, F.; CONTRERAS, P.A; BÖHMWALD, T.M. et al. Efecto de la suplementación con selenio sobre la actividad sanguínea de glutation peroxidasa (GSH-Px) y ganancia de peso en bovinos selenio deficientes mantenidos a pastoreo. Arch. Med. Vet., v. 32 , p.55-62, 2000 .
PAGLIA, D.E.; VALENTINE, W.N. Studies on the quantitative and qualitative characterization of erythrocyte glutathione peroxidase. J. Lab. Clin. Med., v.70, p.158-169, 1967.

PASCHOAL, J.J; ZANETTI, M.A.; DEL CLARO, G.R. et al. Perfil de ácidos graxos e estabilidade oxidativa do leite de vacas holandesas alimentadas com soja extrusada e selênio orgânico. Pesq. Agrop. Bras., v.42, p.1793-1799, 2007.

PHIPPS, R.H.; GRANDISON, A.S.; JONES, A.K. et al. Selenium supplementation of lactating dairy cows: effects on milk production and total selenium content and speciation in blood, milk and cheese. Animal, v.2, p.1610-1618, 2008 .

RAMASWAMY, N.; BAER, R.J.; SCHINGOETHE, D.J. et al. Composition and flavor of milk and butter from cows fed fish oil, extruded soybeans, or their combination. J. Dairy Sci., v.84, p.2144-2151, 2001.

SANTOS, F.L.; SILVA, M.T.C.; LANA, R.P. et al. Efeito da suplementação de lipídios na ração sobre a produção de ácido linoleico conjugado (CLA) e a composição da gordura do leite de vacas. Rev. Bras. Zootec., v.30, p.1931-1938, 2001.

STAGSTED, J. Absence of both glutathione peroxidase activity and glutathione in bovine milk. Internat. Dairy J., v.16, p.662-668, 2006.

SUNDE, R.A.; HOEKSTRA, W.G. Incorporation of selenium from selenite and selenocystine into glutathione peroxidase in the isolated perfused rat liver. Biochem. and Biophysical Rese. Communic., v.93, p.1181-1188, 1980.

SURAI, P.F. Selenium in nutrition and health. 1st ed. United Kingdom: Nottingham University Press, 2006. 974p.

VAN SOEST, P.J; ROBERTSON, J.B.; LEWIS, B.A. Methods for dietary fiber, neutral detergent fiber, and nonstarch polysaccharides in relation to animal nutrition. J. Dairy Sci., v.74, p.3583-3598, 1991.

WARD, A.T.; WITTENBERG, K.M.; PRZYBYISKI, R. Bovine milk fatty profile produced by feeding diets containing solin, flax and canola. J. Dairy Sci., v.85, p.1191-1196, 2002. 